\title{
Active Nonlinear Vibration Absorber Design for Flexible Structures
}

\author{
Lei Chen and Colin H. Hansen ${ }^{\dagger \dagger}$ \\ School of Mechanical Engineering, Adelaide University, SA5005, Australia
}

\author{
Fangpo $\mathrm{He}^{\dagger}$ and Karl Sammut \\ School of Informatics and Engineering, Flinders University, GPO Box 2100, SA5001, Australia
}

(Received 13 July 2006; accepted 11 January 2007)

\begin{abstract}
An active Vibration Clamping Absorber (VCA) technique designed for vibration suppression in flexible structures is proposed and investigated in this paper. The technique uses a Quadratic-Modal-PositivePosition-Feedback strategy to design a simple second-order nonlinear controller that is capable of suppressing structural vibrations at various resonances. The VCA can effectively transfer vibration energy from the main structure to another sacrificial absorber so that large amplitude vibrations in the main structure can be clamped within tolerable limits. The effectiveness of the VCA design is demonstrated through single-mode and multiplemode control on a flexible cantilever beam system using one sensor/actuator pair. The simulation and experimental results reveal that the proposed design can be used for real-time control of vibration in large flexible structures.
\end{abstract}

\footnotetext{
${ }^{\dagger}$ Honorary Fellow of the International Institute of Acoustics and Vibration (IIAV)

${ }^{\dagger}$ Member of the International Institute of Acoustics and Vibration (IIAV)
}

$$
\begin{array}{ll}
\text { Nomenclature } \\
a \quad \text { - vibration amplitude of the beam } \\
b \quad \text { - vibration amplitude of the VCA controller } \\
\mathbf{w}(\mathbf{x}, \mathbf{t}) \text { - displacement of the equilibrium position } \\
v_{k}(t) \quad \text { - modal amplitude of the beam } \\
\omega_{k} \quad \text { - mode frequency of the beam } \\
\phi_{k} \quad \text { - mode shape of the beam } \\
\varphi_{i} \quad \text { - phase angle } \\
\lambda_{k} \quad \text { - eigenvalue of the operator } \Theta \\
\eta(t) \quad \text { - responses of the VCA } \\
\zeta \quad \text { - damping coefficient matrix of the beam } \\
\varpi_{k} \quad \text { - designed natural angular frequency of the VCA } \\
\xi_{k} \quad \text { - VCA controller's damping ratio } \\
G \quad \text { - diagonal gain matrix of the VCA controller } \\
\mathbf{v}_{N} \quad \text { - amplitude vector of the controlled modes } \\
\mathbf{v}_{R} \quad \text { - amplitude vector of the residual modes }
\end{array}
$$

\section{INTRODUCTION}

Flexible beam elements constructed with fabrics, composites, polymers, and light metals are increasingly employed in a variety of large structures in aerospace, robotics, marine, and machinery industries. On one hand, the advance of modern manufacturing technologies has meant that designers can reduce traditional over-design but still produce a reliable and lighter structure. This is often achieved by reducing design safety factors to a minimum, while a traditional structure may be unnecessarily heavy to give the required stiffness and damping to eliminate vibrations. On the other hand, through synthesising techniques, it is possible to use light materials to achieve a high strength-to-weight ratio under stringent constraints of energy consumption. The use of smart structural components that are able to change their stiffness and damping coefficients will enable lighter designs to be achievable.
These lighter structures, however, are physically characterised by low structural damping, low stiffness, and low natural frequencies. Consequently, the structures readily experience high-amplitude resonances under external disturbances, such as forces produced by unbalanced rotating machines, reciprocating machines, or shock impacts. High-amplitude resonances will degrade the reliability and safety of structures. Fatigue failure or even collapse of structures are awesome possibilities under resonant vibration conditions. Therefore, the development of vibration absorbers for flexible structures to reduce low-frequency resonances has been a key area of interest for vibration control of flexible structures.

The difficulties of designing vibration absorbers for flexible structures lie in:

- The primary resonances may be caused by external excitations with time-varying frequencies.

- Multiple harmonic resonances could happen simultaneously.

- The controller's spillover problem may destabilise the control system.

- A simple control algorithm with a low computation load is needed to control both transient and steady state vibrations in real-time.

To solve these problems, various control techniques have been proposed, of which modal control is the most widely reported method. ${ }^{1-4}$ One of the advantages of modal control is that it allows each mode of the structure to be controlled independently of the other modes. Because of this characteristic, standard control problems, such as control system stability and the regulator problem, can be readily addressed.

The history of modal control as applied to vibration suppression can be generally traced back to the 1960 s. $^{1}$ It became popular in the 1970 s and 1980 s but then fell out of favour in the mid 1980s because of the fundamental shortcom- 